\title{
Agnuside Reduces Visceral Adipocyte Size, Improves Insulin Sensitivity and Reverses Hepatic Steatosis in Ovariectomized Rats
}

Franciele Neves Moreno ${ }^{1,2}$, Eduardo Hideo Gilglioni ${ }^{1,2}$, Rosângela Fernandes Garcia ${ }^{3}$, Naiara Cristina Lucredi ${ }^{4}$, Sandra Mara Ferreira ${ }^{5}$, Nilton Almeida Brito ${ }^{3}$, Marcia do Nascimento Brito ${ }^{3}$, Emy Luiza Ishii-Iwamoto ${ }^{1,2}$ and Clairce Luzia Salgueiro-Pagadigorria ${ }^{3}$

1. Laboratory of Experimental Steatosis, Department of Biochemistry, State University of Maringa, Maringa 87020-900, PR, Brazil

2. Laboratory of Biological Oxidations, Department of Biochemistry, State University of Maringa, Maringa 87020-900, PR, Brazil

3. Department of Physiological Sciences, State University of Maringa, Maringa 87020-900, PR, Brazil

4. Laboratory of Biological oxidations, Department of Biochemistry, Federal University of Santa Catarina, Florianopolis 88040-900, SC, Brazil

5. Department of Structural and Functional Biology, Institute of Biology, State University of Campinas (UNICAMP), Campinas 13083-970, SP, Brazil

\begin{abstract}
This study aimed to investigate the effects of agnuside (AGN), a phytoestrogen in Vitex agnus-castus, on cellular and metabolic alterations induced by ovariectomy in rats. Ovariectomized (OVX) Wistar rats were used as an animal model of postmenopausal metabolic syndrome. The visceral and subcutaneous adipocyte sizes, the peripheral insulin sensitivity and the occurrence of nonalcoholic fatty liver disease (NAFLD) and liver oxidative damage were evaluated. The results were compared with those obtained from control rats (sham-operated) and OVX rats treated daily with AGN. Obese OVX rats exhibited visceral adipocyte hypertrophy and developed glucose intolerance, hyperinsulinemia, NAFLD and dyslipidemia. AGN exerted beneficial effects on several metabolic parameters in OVX rats, including the improvement in glucose intolerance, hyperinsulinemia and NAFLD. Besides, AGN promoted a general improvement in the liver redox state. Although the reduction of the mesenteric adipocyte size is very likely to be an important mode of action of AGN, some actions of AGN could also involve the interaction with estrogen receptors.
\end{abstract}

Key words: Metabolic syndrome, ovariectomized rats, adipocyte size, NAFLD, agnuside.

\section{Introduction}

Epidemiological data show that an estimated 1.1 billion women worldwide will be menopausal by 2025 [1]. Postmenopausal women are prone to fat metabolism disorders and obesity, with a central pattern of fat distribution and adipocyte hypertrophy [2], which are both predisposing factors for insulin resistance (IR) [3] and nonalcoholic fatty liver disease

Correspondence: Clairce Luzia Salgueiro-Pagadigorria, Ph.D., professor, research fields: liver, sub cellular fractions, metabolic syndrome, estrogen deficiency and energy metabolism.
(NAFLD) [4, 5]. These features occur because hypertrophic adipocytes exhibit increased lipolysis, releasing fatty acids (FA) directly in the portal venous system. This release overloads the liver and leads to the accumulation of triacylglycerols (TAG) in the cytosol of hepatocytes [6, 7]. Liver lipid metabolism is regulated by estrogens through genomic actions; thus, under conditions of estrogen deficiency, the incidence of NAFLD increases considerably [8]. The fat accumulation in the liver, in turn, leads to oxidative cell damage [9-12].

Hormone replacement therapy has been considered 
the standard treatment for menopausal disturbances. However, the risks associated with this practice for some groups of women $[13,14]$ have led to a search for alternative forms of treatment, including the use of herbal compounds containing phytoestrogens. Although herbal compounds are considered safe, hepatotoxicity induced by active natural compounds is not uncommon, and the alterations induced by estrogen deficiency could make the liver more vulnerable to secondary injuries.

With this in mind, our team has investigated the effects of natural compounds frequently used by postmenopausal women by using ovariectomized (OVX) rats as an animal model $[8,10-12,15,16]$. Approximately 6 weeks after the surgical removal of the ovaries, OVX rats develop several metabolic disturbances characteristic of postmenopausal metabolic syndrome, including increased adiposity, with a central pattern of fat distribution, IR, NAFLD and dyslipidemia [8, 10-12]. Focusing on liver lipid metabolism and toxicity, we demonstrated a high hepatotoxic potential for Cimicifuga racemosa [10], a plant widely used by women to treat menopausal symptoms, especially to relieve hot flushes.

More recently, we investigated the effects of another plant extract, Vitex agnus-castus (VAC), which is also commonly used by postmenopausal women as an alternative therapy [17]. The crude extract of VAC contains considerable quantities of an iridoid glycoside, agnuside (AGN); some of the beneficial effects of VAC have been attributed to the estrogenic and antioxidant actions of AGN [18, 19]. Our results revealed that in OVX rats, the crude extract of VAC and its butanolic fraction (enriched in AGN) are effective in reversing several lipid metabolism disorders, including NAFLD and adiposity [12]. The AGN-enriched extract also exerted additional hepatoprotective effects, including a reduction in the mitochondrial reactive oxygen species (ROS) generation and a general improvement in the redox status. The content of pure AGN in the AGN-enriched extract has been demonstrated to be $8.75 \%$ [12], meaning that other active compounds could also have had a role in the beneficial effects previously found in OVX rats.

Therefore, on basis of the very promising properties of AGN in the treatment of metabolic disturbances associated with estrogen deficiency, this study was planned to investigate the effects of pure AGN on the metabolic disturbances of OVX rats. In addition to liver metabolism, adiposity and the plasma lipid profile, which were previously evaluated after treatment with a crude extract of VAC and an AGN-enriched extract [12] other important parameters related to metabolic syndrome in postmenopausal women were evaluated, including insulin sensitivity, visceral adipocyte size and the formation of advanced glycation end products (AGE).

\section{Materials and Methods}

\subsection{Materials}

AGN and the most of substrates and reagents used were purchased from Sigma Chemical Co. (St. Louis, USA). Some other reagents were from Merck (Darmstadt, FRG), Carlo Erba (São Paulo, Brazil) and Reagen (Rio de Janeiro, Brazil). Gold Analisa ${ }^{\circledR}$ (Belo Horizonte, Brazil) kits for measuring glucose and lipids were used. Sodium heparin was obtained from Roche (São Paulo, Brazil).

\subsection{Animals}

A total of 126 female Wistar rats (45 days old, 130-160 g body weight (BW)) were randomly assigned to receive sham-operation (Control; $\mathrm{CON}$ rats) or bilateral ovariectomy (OVX rats), in a proportion of 1:2. Animals were anaesthetized $(10 \mathrm{mg}$ xylazine + ketamine $50 \mathrm{mg} / \mathrm{kg} \mathrm{BW}$, i.p.) to the surgical removal of the ovaries. The CON rats were subjected to the same procedures without ovary removal. Ten weeks after the ovariectomy or sham-operation surgical procedures, the rats were 
subdivided into three groups: control (sham-operation, CON), untreated OVX rats and AGN-treated ovariectomized rats $(\mathrm{OVX}+\mathrm{AGN})$.

During the entire experimental period of 13 weeks, the rats were maintained in polypropylene cages, at controlled temperature $\left(23^{\circ} \mathrm{C}\right)$ and a light/dark cycle of $12 \mathrm{~h}$. The animals were fed a standard diet and water ad libitum. The food consumption (g/day) and BW were measured in the three groups of animals, throughout the entire experimental period.

All experiments were conducted in strict adherence with the guidelines of the Ethics Committee for Animal Experimentation of the University of Maringá (Certificate No. 149/2014).

\subsection{Animal Treatment and Material Collection}

Ten weeks after the surgical procedures for ovariectomy, the rats of the OVX+AGN group were treated with daily doses of AGN (0.042 mg/kg BW) suspended in gum arabic (1\%). The AGN dose used in this study was equivalent to the amount present in the doses of crude extracts of VAC recommended for postmenopausal women [17]. The CON and OVX (untreated) rats received daily doses of gum arabic $(1 \%)$. The treatment was performed by gavage for a period of 21 days. On the day of the experiments, the animals were anesthetized with thiopental sodium (50 $\mathrm{mg} / \mathrm{kg} \mathrm{BW}$, i.p.) $[10,12]$ and lidocaine $(4 \mathrm{mg} / \mathrm{kg}$ ) [20] to collect blood samples and to remove the liver, adipose tissues and the uterus.

Adipose tissues were collected from three visceral depots (retroperitoneal, mesenteric and uterine) and one subcutaneous depot (inguinal). These adipose tissues were weighed, and the values are expressed in $\mathrm{g}$ per $100 \mathrm{~g}$ of $\mathrm{BW}$. The adiposity index was calculated by adding the weights of these tissues. The uterus was also collected and weighed, and the value was expressed in g per $100 \mathrm{~g} \mathrm{BW}$.

\subsection{Morphometric Analysis of Adipocytes}

The adipocytes were fixed overnight at $4{ }^{\circ} \mathrm{C}$ in
Carnoy's solution (ethanol:chloroform:glacial acetic acid 6:3:1), dehydrated in a graded series of ethanol, diaphanized in xylol and embedded in histological paraffin. The histological tranverse semiserial sections (5 $\mu \mathrm{m}$ thicknesses) were stained with hematoxylin and eosin. Morphometric analyses were carried out using digital images captured by a high resolution camera (Olympus Q Color 3-Olympus American, Burnaby, Canada) coupled to an Olympus BX 41 light microscope with $\times 40$ objectives (Olympus, Tokyo, Japan) using QCapture Pro 5.1 $1^{\mathrm{TM}}$ software. The adipocyte sizes were measured using Image-Pro Plus 4.5 software (Media Cybernetics, Silver Springs, USA) and 400 cells in different microscopic fields per tissue section.

\subsection{Serum Biochemical Analysis}

The blood was collected by cardiac puncture from fasted rats. Total cholesterol, high-density lipoprotein (HDL-cholesterol) and TAG in serum were analyzed by standard methods using assay kits (Gold Analisa ${ }^{\circledR}$ ). The low-density lipoprotein (LDL-cholesterol) levels were determined by subtracting HDL and very-low-density lipoprotein (VLDL-cholesterol) from total cholesterol.

\subsection{Intravenous Glucose Tolerance Test (ivGTT)}

For ivGTT tests, animals fasted for $12 \mathrm{~h}$ were anaesthetized (10 mg xylazine + ketamine $50 \mathrm{mg} / \mathrm{kg}$ BW, i.p.), and a cannula was implanted into the jugular vein for the administration of a glucose load (1 $\mathrm{g} / \mathrm{kg} \mathrm{BW}$ ) and for blood collection during the experiment, which started $24 \mathrm{~h}$ later [21]. The animals were kept awake in individual cages, and blood was collected just before (time 0) and 5, 15 and $30 \mathrm{~min}$ after the injection of the bolus of glucose.

The plasma glucose was measured using assay kits $\left(\right.$ Gold Analisa ${ }^{\circledR}$ ) and insulin by a radioimmunoassay. The fasting insulin and glucose levels were used to evaluate IR, expressed in terms of HOMA-IR index [22]. 


\subsection{Liver Lipid Content Determination}

The total liver lipid content was determined by gravimetry [23], and the lipid contents were expressed in percentage terms $(\mathrm{g} / 100 \mathrm{~g}$ liver wet weight). The liver total cholesterol and TAG were determined after the suspension of fat in $2 \%$ Triton, followed by vortexing and heating at $55^{\circ} \mathrm{C}$. The liver lipid content was measured using assay kits (Gold Analisa ${ }^{\circledR}$ ).

\subsection{Isolation of Liver Fractions and Assays}

Liver mitochondria were isolated by differential centrifugation [24]. Intact mitochondria were used to measure $\beta$-oxidation capacity, ROS generation, protein carbonyl content and nicotinamide nucleotide transhydrogenase (NNT) activity. Freeze-thawing disrupted mitochondria were used to measure the reduced glutathione (GSH) contents, and sonication-disrupted mitochondria were used to measure the activity of glutathione peroxidase 1 (GPx1) [25].

The liver cytosolic fractions were obtained by centrifugation of intact mitochondrial suspensions at $15,000 \times \mathrm{g}$ for $15 \mathrm{~min}$ and were used to measure the activity of the glutathione peroxidase 3 (GPx3).

To measure the activity of the glucose 6-phosphate dehydrogenase (G6PD), livers from fed animals were homogenized in a medium containing $0.1 \mathrm{M}$ Tris/HCl buffer and $1 \mathrm{mM}$ EDTA ( $\mathrm{pH}$ 7.6) and centrifuged at $30,000 \times \mathrm{g}$ for $15 \mathrm{~min}$. The activity of this enzyme was determined in the supernatant by using traditional methods, as described below.

The homogenates obtained from freeze-clamped liver of overnight fasted rats were used to measure GSH and thiobarbituric acid reactive substance (TBARS) levels.

The protein concentrations in the subcellular fractions were determined using bovine serum albumin as a standard [26].

\subsection{Liver Mitochondrial $\beta$-Oxidation Capacity}

The amount of oxygen consumed by intact mitochondria oxidizing FA was measured by polarography at $37{ }^{\circ} \mathrm{C}$ using a Clark-type oxygen electrode (Yellow Springs Instruments, Yellow Springs, OH, USA) [27]. The reactions were initiated by the addition of the following: (a) $20 \mathrm{mM}$ octanoyl-CoA + $2.0 \mathrm{mM}$ L-carnitine, (b) $20 \mathrm{mM}$ palmitoyl-CoA + $2.0 \mathrm{mM}$ L-carnitine or (c) $20 \mathrm{mM}$ palmitoyl-L-carnitine. The rate of oxygen consumption was expressed as $\mathrm{nmol} / \mathrm{min} \times \mathrm{mg}$ protein.

\subsection{Mitochondrial ROS Generation}

The ROS production by the mitochondrial fraction was evaluated using 2',7'-dichlorofluorescein diacetate (DCFH-DA) oxidation assays as previously described [28]. After a 3-min incubation period of the mitochondrial suspensions ( $1 \mathrm{mg}$ protein/mL) in a medium containing $250 \mathrm{mM}$ mannitol, $5 \mathrm{mM}$ malate, $5 \mathrm{mM}$ glutamate, $15 \mu \mathrm{M}$ DCFH-DA and $2 \mathrm{mM}$ TRIS (pH 7.2), the reaction was initiated by the addition of $600 \mu \mathrm{M}$ ADP. The mitochondrial ROS generation was estimated by measuring the linear increase in fluorescence (excitation, $495 \mathrm{~nm}$; emission, $529 \mathrm{~nm}$ ) recorded over a period of $5 \mathrm{~min}$, and the value was expressed as $\mathrm{pmol} / \mathrm{min} \times \mathrm{mg}$ protein.

\subsection{Determination of the GSH Contents in Liver Homogenates and Isolated Mitochondria}

GSH contents were measured in the presence of $o$-phthalaldehyde (OPT) [29], and the product of this reaction, GSH-OPT, was measured fluorimetrically (excitation, $350 \mathrm{~nm}$; emission, $420 \mathrm{~nm}$ ). The results were expressed as $\mu \mathrm{g} / \mathrm{mg}$ protein.

\subsection{Measurements of Antioxidant Enzyme Activities}

The activities of the two isoforms of GPx were assessed in the cytosolic fractions (GPx3) and in the mitochondrial matrix (GPx1) according to their ability to oxidize $\mathrm{GSH}$ in the presence of $\mathrm{H}_{2} \mathrm{O}_{2}$ [30]. The activity of these enzymes was expressed as $\mathrm{nmol} / \mathrm{min}$ $\times \operatorname{mg}$ protein $\left(\varepsilon, 6,220 \mathrm{M}^{-1} \times \mathrm{cm}^{-1}\right)$. 
The activity of G6PD was determined spectrophotometrically by measuring the rate of increasing in absorbance [31] at $340 \mathrm{~nm}$ from the conversion of $\beta$-Nicotinamide adenine dinucleotide $2^{\prime}$-phosphate $\left(\mathrm{NADP}^{+}\right)$to NADPH by G6PD. The activity of this enzyme was expressed in $\mathrm{nmol} / \mathrm{min} \times$ $\mathrm{mg}$ protein $\left(\varepsilon, 6,220 \mathrm{M}^{-1} \times \mathrm{cm}^{-1}\right)$.

The activity of the NNT enzyme was measured in isolated liver mitochondria by direct spectrophotometry using a combination of previously described methods with modifications [32, 33]. The enzyme activity was evaluated by the rate of increase in the absorbance at $375 \mathrm{~nm}$, which was recorded for 3 $\mathrm{min}$; the result was expressed as $\mathrm{mmol} / \mathrm{min} \times \mathrm{mg}$ protein $\left(\varepsilon, 5.1 \mathrm{mM}^{-1} \times \mathrm{cm}^{-1}\right)$.

\subsection{Determination of Protein Carbonyl Content and} Lipid Peroxidation Levels

The protein carbonyl contents were determined in liver homogenates and freshly isolated mitochondria using the 2,4-dinitrophenylhydrazine (DNPH) method described by Guarnier et al. [34] with modifications [35, 36]. The protein carbonyl group levels were evaluated spectrophotometrically at $370 \mathrm{~nm}$; the results were expressed in $\mathrm{nmol} / \mathrm{mg}$ protein $\left(\varepsilon, 22,000 \mathrm{M}^{-1} \times \mathrm{cm}^{-1}\right)$.

The TBARS contents (predominantly malondialdehyde) were measured by direct spectrophotometry [37] and used as biomarkers of oxidative stress and lipid peroxidation. The results were expressed as nmol/mg protein $\left(\varepsilon, 1.56 \times 10^{5}\right.$ $\left.\mathrm{M}^{-1} \times \mathrm{cm}^{-1}\right)$.

\subsection{Treatment of Data}

The data in the figures and tables are presented as mean \pm standard error (SE) and were subjected to analysis of normality using the Shapiro-Wilk test. The experimental groups were compared using analysis of variance (ANOVA), followed by Newman-Keuls post hoc test. The compared values are provided in the text as probability values $(p)$, and the minimum criterion of significance was $p \leq 0.05$. Statistical analyses were performed with GraphPad Prism 5.0 software (GraphPad Software, Inc.).

\section{Results}

\subsection{General Features}

As shown in the graph line in Fig. 1, the OVX and $\mathrm{OVX}+\mathrm{AGN}$ rats exhibited a higher and similar BW gain (approximately 60\%), compared to the CON group. Fig. 1 also shows a histogram of the BW gain at end of the experimental period.

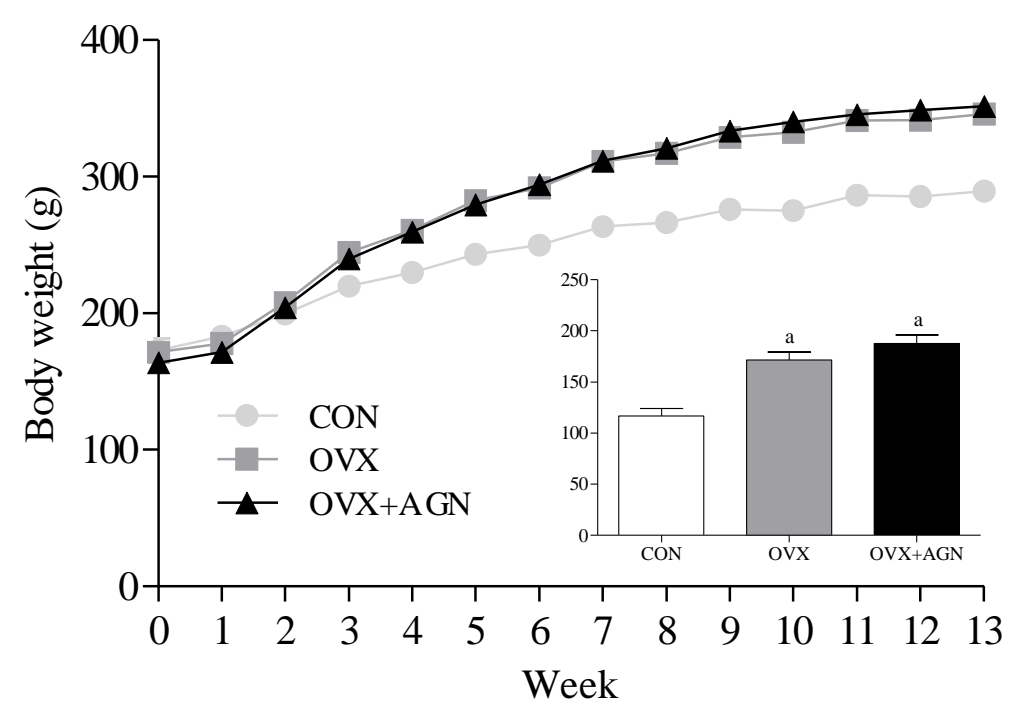

Fig. 1 Body weight evolution. The CON, OVX and OVX+AGN rats were weighed weekly $(n=7)$. The graph line shows the body weight (g) over the time (13 weeks). The histogram represents the body weight gain of each group at the end of the experimental period. The results were expressed as mean $\pm \mathrm{SE}\left({ }^{\mathrm{a}} p<0.001\right.$ vs. CON $)$. 
Table 1 presents the general features of these animals. Among them, the uterine weight, which reveals uterine atrophy in the OVX and OVX+AGN rats, indicates estrogen deficiency. Although the OVX and $\mathrm{OVX}+\mathrm{AGN}$ rats exhibited higher BW gain than $\mathrm{CON}$ rats, this could not be attributed to differences in food ingestion. The adiposity index was higher only in OVX rats $(+29 \%)$. In these animals, with the exception of retroperitoneal fat, all other fat depots increased compared to those in $\mathrm{CON}$ : mesenteric by $55 \%$, uterine by $25 \%$ and inguinal fat by $35 \%$. The treatment with AGN was effective in reducing the mesenteric and uterine fat depots, which became similar to those from CON rats, but this treatment failed to reduce the inguinal fat $(+32 \%)$. In the $\mathrm{OVX}+\mathrm{AGN}$ rats, the adiposity index reached intermediate values between those found in the CON and OVX rats but did not differ significantly from those values.

\subsection{Morphometric Analysis of Adipocytes}

Fig. 2 shows the representative photomicrographs of the retroperitoneal (panels $\mathrm{A}, \mathrm{B}, \mathrm{C}$ ), mesenteric (panels D, E, F), uterine (G, H, I) and inguinal (J, K, L) fat depots from the CON, OVX and OVX+AGN rats, respectively. Panels 1a, 1b, 1c and 1d show the average adipocyte sizes in these depots. The adipocyte sizes were significantly higher in the OVX rats, except in the retroperitoneal fat. The treatment with AGN reduced the adipocyte sizes in the mesenteric and uterine fat depots; these adipocyte sizes became similar to those of the CON rats. However, the treatment was not effective in reducing the adipocyte sizes in the inguinal fat.

\subsection{Serum Biochemical Analysis}

Table 2 shows the lipid profile of these animals. The TAG and total cholesterol were directly measured by biochemical methods. As the animals of all groups presented values of total cholesterol lower than 100 $\mathrm{mg} / \mathrm{dL}$, the Friedewald formula could be used reliably to calculate the VLDL-cholesterol levels [38]. Compared with the CON rats, the OVX rats presented higher TAG and VLDL levels (approximately 29\% higher for both). The treatment with AGN further increased the TAG and VLDL serum levels, which were both $53 \%$ higher than those of CON. The total, HDL-, and LDL-cholesterol levels did not differ between the groups; neither did the HDL/LDL ratio.

\subsection{Intravenous Glucose Tolerance Test (ivGTT)}

Fig. 3 shows the time course of glucose (panel A) and insulin (panel B) levels. Although the glycemic responses of the animals did not differ between groups, the insulin peak at $5 \mathrm{~min}$ was significantly higher in OVX rats than in CON and OVX+AGN rats. These results were corroborated by the histograms of the areas under the curves (AUCs) of glucose (panel C) and insulin (panel D) levels. The HOMA-IR index (panel E) did not differ between the groups.

Table 1 General features.

\begin{tabular}{llll}
\hline & CON & OVX & OVX+AGN \\
\hline Uterine weight & $0.25 \pm 0.02$ & $0.04 \pm 0.01^{\mathrm{a}}$ & $0.04 \pm 0.01^{\mathrm{a}}$ \\
Food consumption & $20.21 \pm 0.42$ & $21.90 \pm 0.38$ & $21.59 \pm 0.89$ \\
Adiposity index & $7.88 \pm 0.47$ & $10.23 \pm 0.75^{\mathrm{b}}$ & $9.39 \pm 0.20$ \\
Retroperitoneal fat & $2.37 \pm 0.24$ & $2.28 \pm 0.16$ & $2.33 \pm 0.10$ \\
Mesenteric fat & $1.42 \pm 0.11$ & $2.20 \pm 0.19^{\mathrm{c}}$ & $1.76 \pm 0.07$ \\
Uterine fat & $2.15 \pm 0.14$ & $2.68 \pm 0.15^{\mathrm{c}}$ & $2.14 \pm 0.07$ \\
Inguinal fat & $2.06 \pm 0.16$ & $2.78 \pm 0.12^{\mathrm{d}}$ & $2.72 \pm 0.14^{\mathrm{d}}$ \\
\hline
\end{tabular}

The uterine and fat depot weights were expressed in g/100 g BW $(n=6-8)$. Food consumption was expressed in g/day $(n=3)$. The adiposity index was expressed as $\mathrm{g} / 100 \mathrm{~g} \mathrm{BW}(n=6)$. The results were expressed as mean $\pm \mathrm{SE}\left({ }^{\mathrm{a}} p<0.001 \mathrm{vs}\right.$.CON; ${ }^{\mathrm{b}} p<0.05 \mathrm{vs}$. CON; ${ }^{c} p<0.05$ vs. CON and OVX+AGN; ${ }^{\mathrm{d}} p<0.01$ vs. CON). 


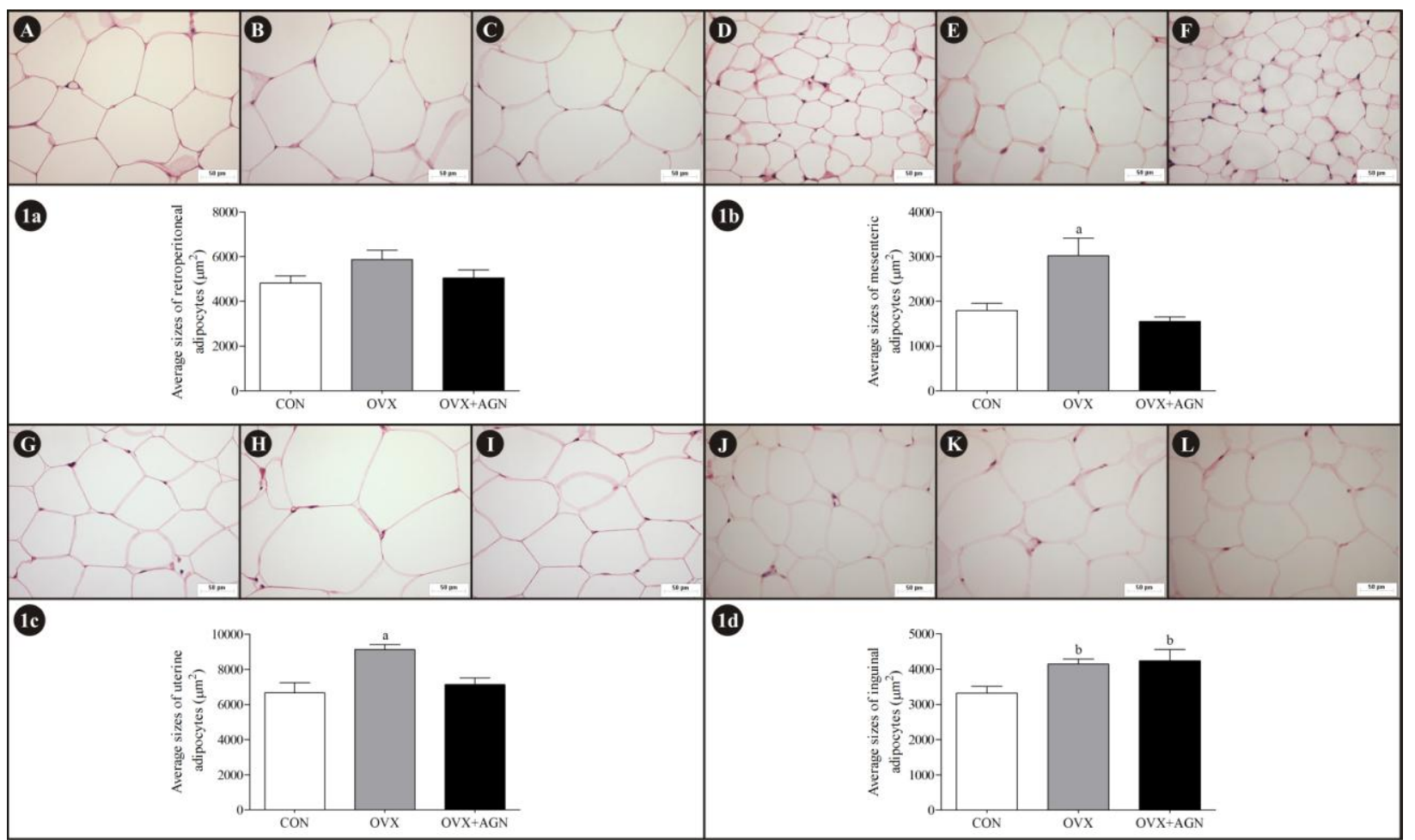

Fig. 2 Adipocyte size of retroperitoneal, mesenteric, uterine and inguinal fat depots. Representative photomicrographs of adipose tissues of CON, OVX and OVX+AGN rats, from retroperitoneal (panels A, B, C, respectively), mesenteric (panels D, $\mathrm{E}, \mathrm{F}$ ), uterine (panels $\mathrm{G}, \mathrm{H}, \mathrm{I}$ ) and inguinal $(\mathrm{J}, \mathrm{K}, \mathrm{L}$ ) fat depots. Below these photomicrographs, are presented the morphometric analysis of the adipocyte size from CON, OVX and OVX+AGN rats, performed for all these fat depots (panel 1a, $n=5$; panel 1b, $n=4$; panel 1c, $n=5$ and panel 1d, $n=5$ ). The scale bar is of $50 \mu \mathrm{m}$. The results were expressed as mean \pm SE ( ${ }^{a} p<0.05$ vs. CON and OVX+AGN; ${ }^{b} p<0.05$ vs. CON).

Table 2 Serum biochemical analysis.

\begin{tabular}{llll}
\hline & CON & OVX & OVX+AGN \\
\hline Triacylglycerols & $31.58 \pm 2.29$ & $40.62 \pm 2.04^{\mathrm{a}}$ & $48.29 \pm 1.79^{\mathrm{b}}$ \\
Total cholesterol & $65.67 \pm 3.61$ & $72.12 \pm 2.86$ & $70.86 \pm 1.62$ \\
HDL-cholesterol & $32.15 \pm 2.88$ & $35.11 \pm 1.59$ & $34.14 \pm 1.75$ \\
LDL-cholesterol & $28.50 \pm 2.24$ & $30.44 \pm 1.97$ & $26.64 \pm 1.58$ \\
VLDL-cholesterol & $6.32 \pm 0.46$ & $8.12 \pm 0.41^{\mathrm{a}}$ & $9.66 \pm 0.36^{\mathrm{b}}$ \\
HDL/LDL ratio & $1.17 \pm 0.14$ & $1.18 \pm 0.16$ & $1.43 \pm 0.16$ \\
\hline
\end{tabular}

Triacylglycerols $(\mathrm{mg} / \mathrm{dL} ; n=12)$, total cholesterol $(\mathrm{mg} / \mathrm{dL} ; n=7)$, high-density lipoprotein (HDL-cholesterol; $\mathrm{mg} / \mathrm{dL} ; n=7$ ), low-density lipoprotein (LDL-cholesterol; $\mathrm{mg} / \mathrm{dL} ; n=6$ ), very low density lipoprotein (VLDL-cholesterol; $\mathrm{mg} / \mathrm{dL} ; n=12$ ) and HDL/LDL ratio $(n=5)$ were expressed as mean $\pm \mathrm{SE}\left({ }^{\mathrm{a}} p<0.05\right.$ vs. CON; ${ }^{\mathrm{b}} p<0.05$ vs. CON and OVX).

\subsection{Liver Lipid Content Determination}

To evaluate the occurrence of NAFLD, the total liver lipid contents were measured (Fig. 4A); the livers of $\mathrm{CON}$ rats presented a normal total lipid content ( $4.39 \pm 0.27 \mathrm{~g} / 100 \mathrm{~g}$ liver wet weight), while the livers of OVX rats exhibited significantly higher amount of total lipids $(+38 \%)$, indicating extensive NAFLD. This condition was partially reversed by treatment with AGN. In the OVX+AGN rats, the liver lipid contents were between the values in the OVX and $\mathrm{CON}$ rats and differed significantly from the values in those groups.

The amounts of TAG (Fig. 4B) and total cholesterol (Fig. 4C) in the liver were also quantified. The TAG contents were higher in the OVX rats $(+40 \%)$ than in the $\mathrm{CON}$ rats, and the treatment reduced these values to close to those found in CON. The amount of total 

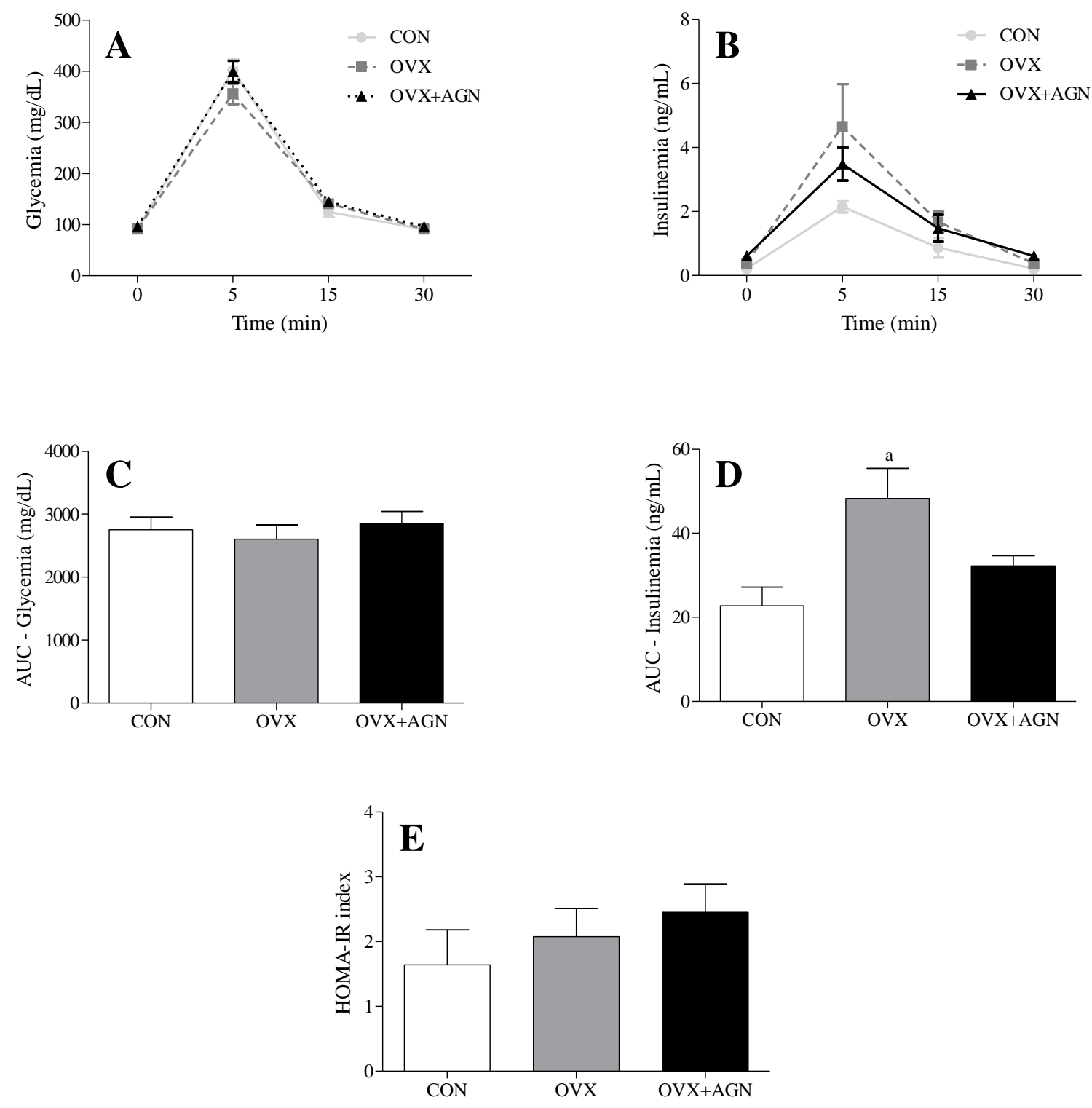

Fig. 3 Plasma glucose and insulin concentrations from intravenous glucose tolerance test (ivGTT). Time course of glycemia (Panel A, mg/dL; $n=7-8$ ), time course of insulinemia (Panel B, $\mathrm{ng} / \mathrm{mL} ; \boldsymbol{n}=5-6$ ), the areas under the curves (AUC) of glycemia (panel C) and insulinemia (panel D), and HOMA-IR index (Panel E, $n=5-6$ ) are presented. The results were expressed as mean $\pm \mathrm{SE}\left({ }^{\mathrm{a}} p<0.05\right.$ vs. CON and OVX+AGN). 

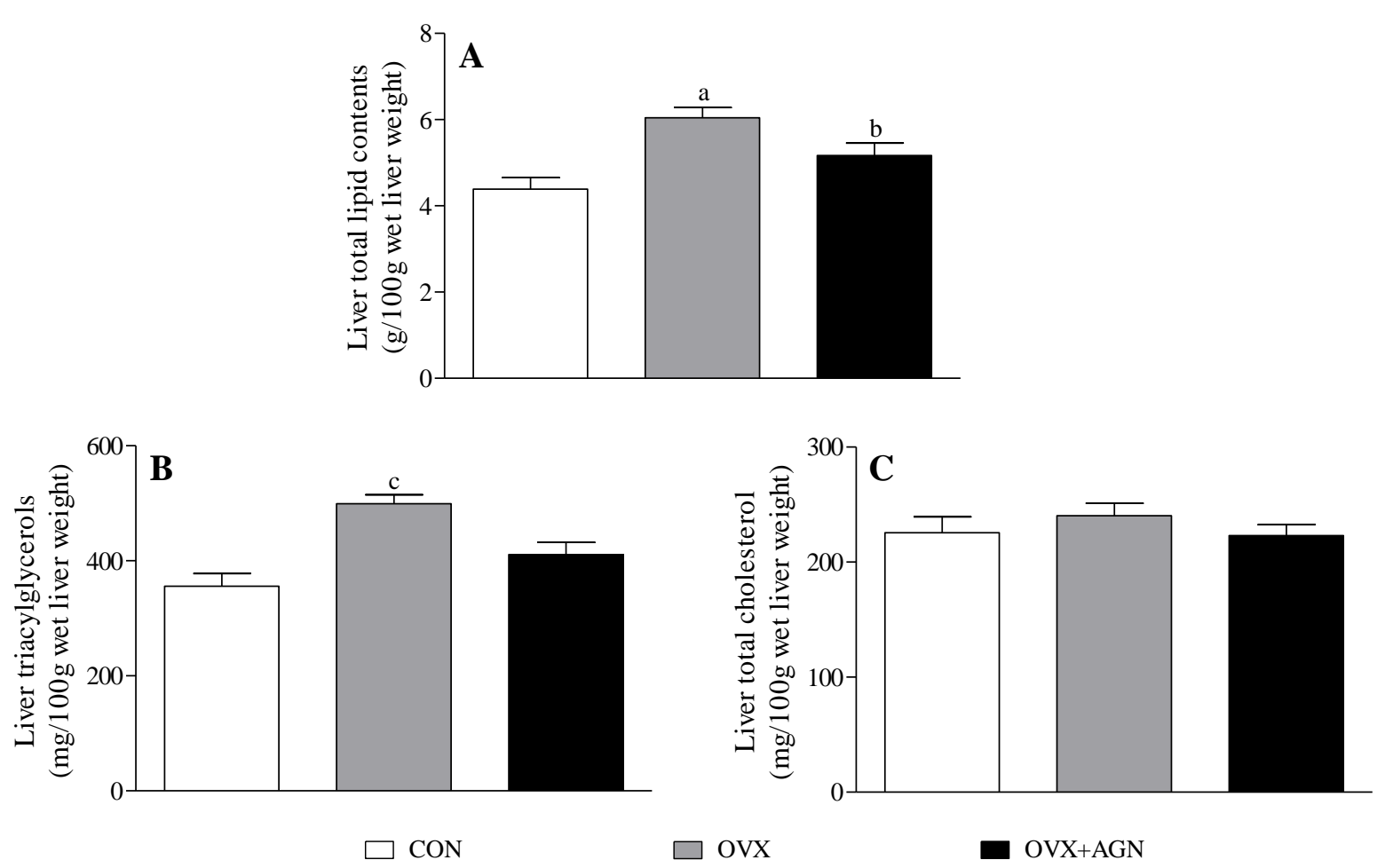

Fig. 4 Liver lipid content. The liver total lipid content (Panel A, g/100 g wet liver weight; $n=14$ ), the liver TAG (Panel B, $\mathrm{mg} / 100 \mathrm{~g}$ wet liver weight; $\boldsymbol{n}=\mathbf{1 3})$ and in the liver total cholesterol (Panel $\mathrm{C}, \mathrm{mg} / 100 \mathrm{~g}$ wet liver weight; $n=12)$ are presented. The results were expressed as mean \pm SE $\left({ }^{a} p<0.01\right.$ vs. CON; ${ }^{b} p<0.05$ vs. CON and OVX; ${ }^{c} p<0.05$ vs. CON and OVX+AGN).

cholesterol in the liver was not different between groups.

\subsection{Liver Mitochondrial $\beta$-Oxidation Capacity}

The ability of isolated liver mitochondria to oxidize octanoyl- and palmitoyl-CoA (in the presence of L-carnitine) and palmitoyl-L-carnitine was measured, and the results are shown in Fig. 5. The oxidation of octanoyl-CoA and palmitoyl-L-carnitine in isolated liver mitochondria was not different between groups, but less oxidation of palmitoyl-CoA was found in the $\mathrm{OVX}$ and $\mathrm{OVX}+\mathrm{AGN}$ rats than in the $\mathrm{CON}$ rats.

\subsection{Liver Mitochondrial Redox State}

Mitochondrial ROS generation and the activity of mitochondrial antioxidant enzymes were also evaluated in this work, and the results are shown in Fig. 6. As shown in Panel A, the liver mitochondrial $\mathrm{H}_{2} \mathrm{O}_{2}$ production from $\mathrm{OVX}$ rats was significantly higher than that of the CON rats $(+238 \%)$, and the treatment partially reduced this production by approximately 30\%. Panel B shows the carbonyl protein contents in mitochondria, which were higher in the OVX rats $(+98 \%)$ than in the CON rats and were completely restored in the OVX+AGN rats. The mitochondrial GSH contents, which were reduced in the OVX rats (Panel C), were not significantly restored in the OVX+AGN rats. The activities of the following mitochondrial antioxidant enzymes were also assessed in isolated mitochondria: GPx1 (Panel D) and NNT (Panel E). Similar results were found for these two enzymes: both were lower in the liver mitochondria of the OVX rats than in those of the CON rats (-28\% and $-29 \%$, respectively). The treatment with AGN completely restored the GPx1 activity, but did not significantly alter the NNT activity, which reached intermediate values between CON and OVX rats, but did not differ to them. 


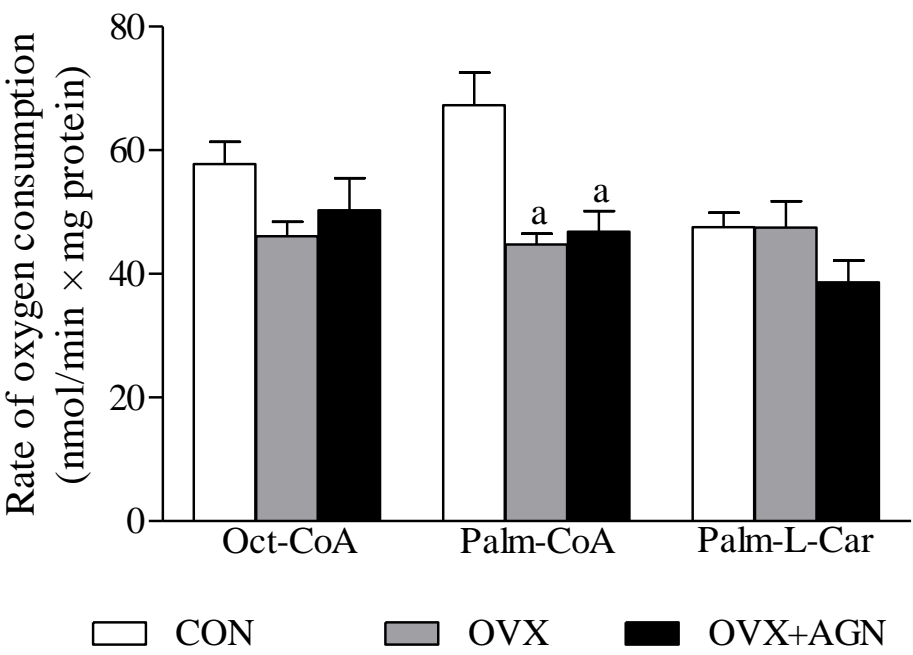

Fig. 5 Liver mitochondrial $\beta$-oxidation. The liver mitochondrial $\beta$-oxidation capacity in rats was determined by polarography as described in the Material and Methods section. Reactions were initiated by the addition of the octanoyl-CoA + L-carnitine (Oct-CoA), palmitoyl-CoA + L-carnitine (Palm-CoA) or palmitoyl-L-carnitine (Palm-L-Carn). The values are expressed as the means of 3 to 6 individual experiments with different mitochondrial preparations $(\mathrm{nmol} / \mathrm{min} \times \mathrm{mg}$ protein). The results were expressed as mean $\pm \mathrm{SE}\left({ }^{\mathrm{a}} p<0.05\right.$ vs. CON $)$.
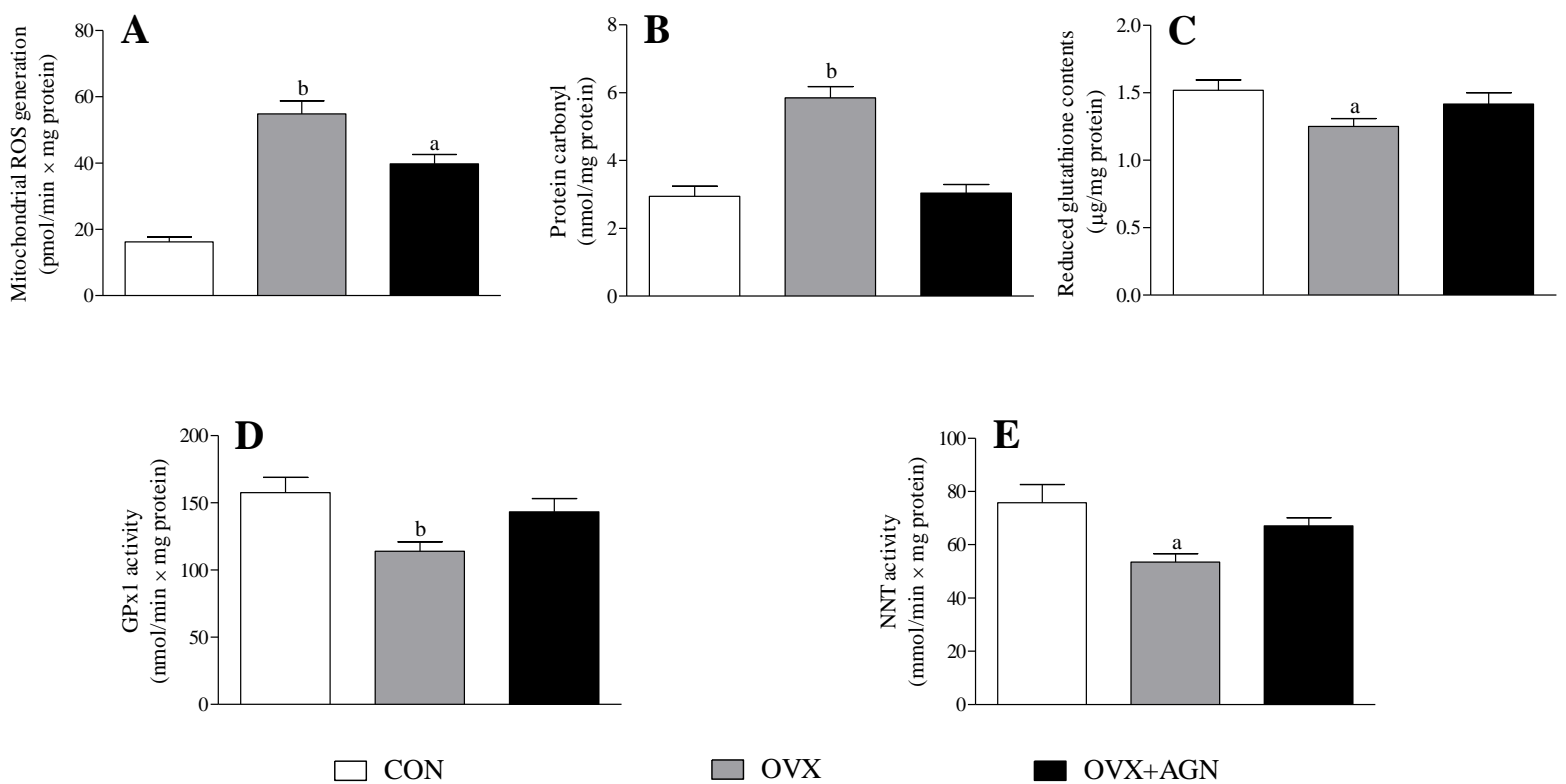

Fig. 6 Mitochondrial ROS generation and ROS scavenger systems. The liver mitochondrial oxidative status was evaluated by assessing mitochondrial ROS generation and the ROS scavenging system. Mitochondrial $\mathrm{H}_{2} \mathrm{O}_{2}$ generation (Panel A, $\mathrm{pmol} / \mathrm{min} \times \mathrm{mg}$ protein; $\boldsymbol{n}=\mathbf{5}$ ); mitochondrial protein carbonyl (Panel B, nmol/mg protein; $\boldsymbol{n}=\mathbf{8}$ ); mitochondrial GSH levels (Panel C, $\mu \mathrm{g} / \mathrm{mg}$ protein; $\boldsymbol{n}=8$ ); GPx1 activity (Panel D, nmol $/ \mathrm{min} \times \mathrm{mg}$ protein; $\boldsymbol{n}=8$ ) and NNT activity (Panel E, mmol/min $\times \mathrm{mg}$ protein; $n=6$ ) were evaluated. The results were expressed as mean $\pm \mathrm{SE}\left({ }^{\mathrm{a}} p<0.05\right.$ vs. CON; ${ }^{\mathrm{b}} p<0.05$ vs. CON and OVX+AGN). 

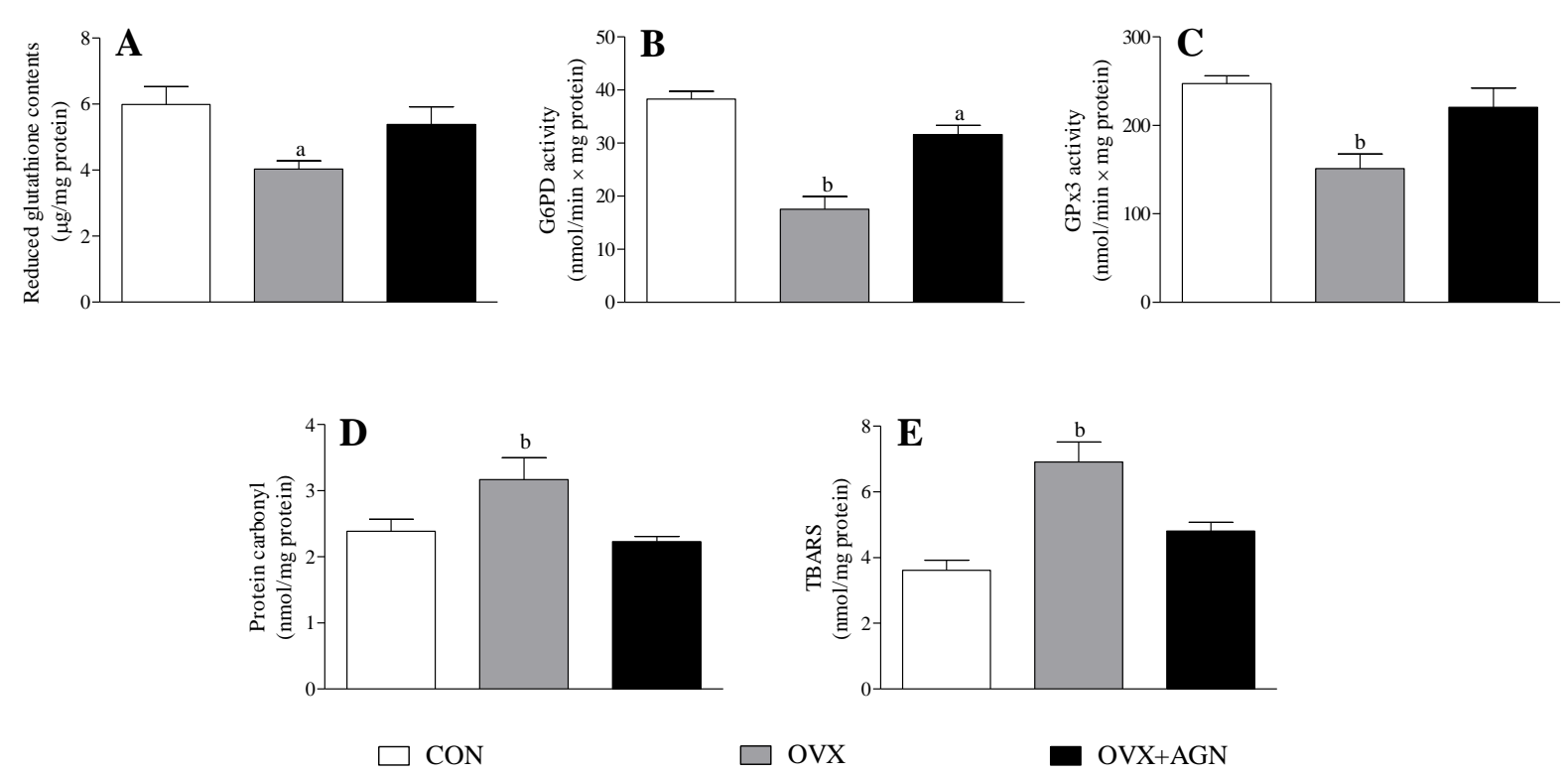

Fig. 7 Evaluation of the liver redox status. The liver oxidative status was evaluated by assessing the liver GSH levels (Panel A, $\mu \mathrm{g} / \mathrm{mg}$ protein; $n=7$ ); G6PD activity (Panel B, nmol/min $\times$ mg protein; $n=7$ ); GPx3 activity (Panel C, $\mathrm{nmol} / \mathrm{min} \times \mathrm{mg}$ protein; $n=9$ ); protein carbonyl (Panel D, nmol/mg protein; $n=7$ ) and liver lipid peroxidation using the TBARS method (Panel E, nmol/mg protein; $n=8$ ). The results were expressed as mean $\pm \mathrm{SE}\left({ }^{\mathrm{a}} p<0.05\right.$ vs. CON; ${ }^{\mathrm{b}}<<0.05$ vs. CON and OVX+AGN).

\subsection{Liver Redox State}

The increased ROS generation found in the liver mitochondria from the OVX rats could induce alterations in the general redox state, a possibility that was evaluated in this work through GSH and TBARS measurements. The activities of cytosolic antioxidant enzymes were also measured, and the results are presented in Fig. 7 (panels A to E). The GSH levels (panel A) were significantly reduced in the OVX rats $(-33 \%)$ and were not significantly restored by treatment with AGN. The level of G6PD enzyme, (panel B), which provides the reducing equivalents to glutathione reductase, was reduced $(-54 \%)$ in the OVX rats and was partially recovered by treatment. The GPx3 activities (panel C) were reduced (-39\%) in the OVX rats and were completely restored in the OVX+AGN rats. The carbonyl protein content in the liver homogenate (panel D) was significantly higher $(+33 \%)$ in the OVX rats and was completely recovered in the $\mathrm{OVX}+\mathrm{AGN}$ rats; similar results were found for the lipid peroxidation levels (panel E).

\section{Discussion and Conclusions}

The results presented here demonstrated that the OVX rats exhibited increases in almost all fat depots and in the adiposity index. The adipocytes in two visceral depots (mesenteric and uterine) became hypertrophic in OVX rats, a phenomenon that was accompanied by glucose intolerance, NAFLD and dyslipidemia. Additionally, fat in the liver led to worsening of the general redox state of this organ. The treatment of OVX rats with AGN reduced the visceral adipocyte size, improved the glucose tolerance, reduced the liver fat accumulation and improved the liver redox state.

Among all the adipocytes, the mesenteric adipocytes in the CON rats exhibited the smallest diameters in the CON rats, and the mesenteric adipocytes in the OVX rats exhibited the largest size increases. On the other hand, the largest adipocytes of the CON rats either did not become hypertrophic (retroperitoneal depot) or exhibited a small increase in size (subcutaneous depot). These same features were 
reported for comparisons of lean premenopausal and obese postmenopausal women [2], as well as for comparisons of healthy and unhealthy, severely obese men [3].

The treatment of OVX rats with AGN was able to reduce the adiposity index and the size of visceral adipocytes, particularly the mesenteric ones, without affecting the diameter of the subcutaneous adipocytes, and this effect was accompanied by improvements in glucose tolerance and NAFLD, which corroborates the correlation between mesenteric adipocyte hypertrophy and metabolic disorders.

Omental/mesenteric adipocyte hypertrophy leads to more detrimental metabolic effects, in part because of the close proximity to the liver and visceral immune cell populations [7]. In addition, the enlarged visceral adipocytes exhibit the most pronounced diameter-related alterations, including high tumor necrosis factor (TNF- $\alpha$ ) [39] and low adiponectin levels [40], which were shown to be related to lower responsiveness of adipocytes to insulin [41, 42] thus resulting in increased adipocyte lipolysis [43] and less glucose uptake [44].

Although the alterations observed in OVX rats occurred in the absence of significant changes in insulin sensitivity, as judged by the HOMA-IR index, under conditions of glucose overload, the OVX rats presented higher insulin peaks, suggesting reduced insulin sensitivity.

The treatment of OVX rats with AGN led to an improvement in insulin sensitivity, and this improvement could contribute to the partial reversal of NAFLD, partly because of the lower lipolytic response of mesenteric adipocytes [7] and partly because of improvements in hepatic lipid metabolism [45]. The latter effect seems to be related to the estrogen-like actions of AGN [8]. The reduction in insulin levels by AGN could also contribute to higher VLDL secretion, since insulin promotes autophagic degradation of apolipoprotein B100 (apoB100), thus limiting secretion of VLDL from the liver [15]. On the one hand, this change could help reverse NAFLD, but on the other hand, it could provoke elevations in VLDL levels, which, in fact, was a finding of the present work in OVX rats treated with AGN.

With respect to the lipid profile, the fasting VLDL and TAG levels were increased in the OVX rats and were further increased by the treatment with AGN. However, we believe that the mechanisms involved are quite different. In OVX rats, the high levels of VLDL could probably be a consequence of the physiological loss of the hepatic insulin regulation of apoB100 that resulted from the liver fat accumulation [46]. In the OVX+AGN animals, the increase in the VLDL levels should reflect higher liver secretion of VLDL. Similar results were found upon treatment of OVX rodents with estrogen $[15,16]$.

The liver redox state of these animals was also evaluated in this study. OVX rats exhibited a general worsening of the liver redox state. It is known that liver fat accumulation per se leads to oxidative cell damage [9]. In addition, estrogen has antioxidant actions not only because of its phenolic structure [47] but also because of its capacity to decrease mitochondrial ROS generation [48] and to regulate the expression or activity of antioxidant enzymes [10-12, 47, 48].

Among the beneficial effects of AGN on redox status, the complete restoration of the activities of GPx1, Gpx3 and G6PD stands out. The partial recovery of the NNT activity upon AGN treatment could be a result of the reduction of TAG in the liver of OVX+AGN rats, since NNT is strongly inhibited by FA, primarily long chain FA (palmitoyl-CoA) [49, 50]. It is also reasonable to consider that the partial recovery of the activities of the NNT and G6PD enzymes could have contributed to the partial restoration of the GSH levels. For the activities of two other important antioxidant enzymes, superoxide dismutase and catalase, in our previous study [12], we found that their activities were not altered by ovariectomy, so these enzymes were not studied in 
this work.

In summary, our results support the existence of a close relationship between mesenteric adipocyte hypertrophy and glucose intolerance, hyperinsulinemia and NAFLD in OVX rats. The reduction in the mesenteric adipocyte size is very likely an important mode of action of AGN. The actions of AGN could also involve interactions with estrogen receptors, as indicated by the increase in VLDL secretion [15], the reduction in ROS generation [48] and the overall improvement in the liver redox state.

Our findings reveal that AGN cannot be considered the main active compound in VAC, as has been suggested [17], since the treatment of OVX rats with pure AGN could not completely reverse the hepatic steatosis and adiposity index, as previously observed for an AGN-enriched extract of VAC [12]. This finding corroborated the idea that other active compounds in the VAC extracts could act synergistically, as is very common in herbal medicines. Nevertheless, the high potential for the use of pure AGN to treat postmenopausal-related metabolic disturbances was evidenced by our current study, especially the improvement in insulin resistance and the reduction in the visceral adipocyte size.

\section{Financial Support}

This work was supported by the Coordenação de Aperfeiçoamento de Pessoal de Nível Superior (Capes) and Conselho Nacional de Desenvolvimento Científico e Tecnológico (CNPq).

\section{Conflicts of Interest}

The authors declare no conflict of interest.

\section{Acknowledgments}

We acknowledge Doctor Maria Raquel Marçal Natali for having authorized the development of some stages of this study in your laboratory and the technical support granted, and the student Gustavo
Felipe Guandalin for the contribution in the maintenance of the animals and in some experiments.

\section{Reference}

[1] Brady, C. W. 2015. "Liver Disease in Menopause." World J. Gastroenterol. $21 \quad$ (25): 7613-20. https://doi.org/10.3748/wjg.v21.i25.7613.

[2] Santosa, S., and Jensen, M. D. 2013. “Adipocyte Fatty Acid Storage Factors Enhance Subcutaneous Fat Storage in Postmenopausal Women.” Diabetes 62 (3): 775-82. https://doi.org/10.2337/db12-0912.

[3] O’Connell, J., Lynch, L., Cawood, T. J., Kwasnik, A., Nolan, N., Geoghegan, J., et al. 2010. "The Relations Hip of Omental and Subcutane ous Adipocyte Size to Metabolic Disease in Severe Obesity." PLoS ONE 5 (4): e9997. https://doi.org/10.1371/journal.pone.0009997.

[4] Carr, M. C. 2003. "The Emergence of the Metabolic Syndrome with Menopause." J. Clin. Endocrinol. Metab. 88: 2404-11. https://doi.org/10.1210/jc.2003-030242.

[5] Völzke, H., Schwarz, S., Baumeister, S. E., Wallaschofski, H., Schwahn, C., Grabe, H. J., et al. 2007. "Menopausal Status and Hepatic Steatosis in a General Female Population." Gut 56: 594-5. https://doi.org/10.1136/gut.2006.115345.

[6] Wajchenberg, B. L. 2000. "Subcutaneous and Visceral Adipose Tissue: Their Relation to the Metabolic Syndrome." Endocr. Rev. 21 (6): 697-738. https://doi.org/10.1210/edrv.21.6.0415.

[7] Yang, Y. K., Chen, M., Clements, R. H., Abrams, G. A., Aprahamian, C. J., and Harmon, C. M. 2008. "Human Mesenteric Adipose Tissue Plays Unique Role versus Subcutaneous and Omental Fat in Obesity Related Diabetes." Cell Physiol. Biochem. 22: 531-8. https://doi.org/10.1159/000185527.

[8] Paquette, A., Wang, D., Jankowski, M., Gutkowska, J., and Lavoie, J. M. 2008. "Effects of Ovariectomy on PPAR Alpha, SREBP-1c, and SCD-1 Gene Expression in the Rat Liver." Menopause 15: 1169-75. https://doi.org/10.1097/gme.0b013e31817b8159.

[9] Ibrahim, S. H., Kohli, R., and Gores, G. J. 2011. "Mechanisms of Lipotoxicity in NAFLD and Clinical Implications.” J. Pediatr. Gastroenterol. Nutr. 53: 131-40. https://doi.org/10.1097/MPG.0b013e31822578db.

[10] Campos, L. B., Gilglioni, E. H., Garcia, R. F., Brito, M. N., Natali, M. R., Ishii-Iwamoto, E. L., et al. 2012. "Cimicifuga racemosa Impairs Fatty acid $\beta$-Oxidation and Induces Oxidative Stress in Livers of Ovariectomized Rats with Renovascular Hypertension.” Free Radic. Biol. Med. 15: 680-9. https://doi.org/10.1016/j.freeradbiomed.2012.05.043. 
[11] Gilglioni, E. H., Campos, L. B., Oliveira, M. C., Garcia, R. F., Ambiel, C. R., Buzzo, A. J., et al. 2013. "Beneficial Effects of Tibolone on Blood Pressure and Liver Redox Status in Ovariectomized Rats with Renovascular Hypertension." J. Gerontol. A. Biol. Sci. Med. Sci. 68: 510-20. https://doi.org/10.1093/gerona/gls210.

[12] Moreno, F. N., Campos-Shimada, L. B., Costa, S. C., Garcia, R. F., Cecchini, A. L., Natali, M. R. M., et al. 2015. "Vitex agnus-castus L. (Verbenaceae) Improves the Liver Lipid Metabolism and Redox State of Ovariectomized Rats." Evid. Based. Complement. Alternat. $\quad$ Med. $\quad 212378$ : https://doi.org/10.1155/2015/212378.

[13] Manson, J. E. 2013. "The Role of Personalized Medicine in Identifying Appropriate Candidates for Menopausal Estrogen Therapy." Metabolism 62 (1): S15-9. https://doi.org/10.1016/j.metabol.2012.08.015.

[14] Bassuk, S. S., and Manson, J. E. 2016. "The timing hypothesis: Do coronary risks of menopausal hormone therapy vary by age or time since menopause onset?" $\begin{array}{llll}\text { Metabolism } & 65 & \text { (5): } & \text { 794-803. }\end{array}$ https://doi.org/10.1016/j.metabol.2016.01.004.

[15] Zhu, L., Brown, W. C., Cai, Q., Krust, A., Chambon, P., McGuinnes, O. P., et al. 2013. "Estrogen Treatment after Ovariectomy Protects against Fatty Liver and May Improve Pathway-Selective Insulin Resistance." Diabetes 62: 424-34. https://doi.org/10.2337/db11-1718.

[16] Nigro, M., Santos, A. T., Brathem, C. S., Louzada, R. A. N., Fortunato, R. S., Ketzer, K. D. P., et al. 2014. "A Change in Liver Metabolism but Not in Brown Adipose Tissue Thermogenesis Is an Early Event in Ovariectomy-Induced Obesity in Rats." Endocrinology 155 (8): 2881-91. https://doi.org/10.1210/en.2013-1385.

[17] Schellenberg, R., Zimmermann, C., Dreweb, J., Hoexterc, G., and Zahnerb, C. 2012. "Dose-Dependent Efficacy of the Vitex agnus castus Extract Ze 440 in Patients Suffering from Premenstrual Syndrome." Phytomedicine 19:

1325-31. https://doi.org/10.1016/j.phymed.2012.08.006.

[18] Hu, Y., Hou, T. T., Zhang, Q. Y., Xin, H. L., Zheng, H. C., Rahman, K., et al. 2007. "Evaluation of the Estrogenic Activity of the Constituents in the Fruits of Vitex rotundifolia L. for the Potential Treatment of Premenstrual Syndrome." J. Pharm. Pharmacol. 59 (9): 1307-12. https://doi.org/10.1211/jpp.59.9.0016.

[19] Kadir, F. A., Kassim, N. M., Abdulla, M. A., and Yehye, W. A. 2013. "PASS-Predicted Vitex negundo Activity: Antioxidant and Antiproliferative Properties on Human Hepatoma Cells-An in Vitro Study." BMC Complementary and Altern. Med. 13: 343. https://doi.org/10.1186/1472-6882-13-343.

[20] Sharp, P., and Villano, J. S. 2013. The Laboratory
Rat. 2nd ed. CRC Press, Taylor \& Francis Group, Boca Raton.

[21] Harms, P. G., and Ojeda, S. R. 1974. "A Rapid and Simple Procedure for Chronic Cannulation of the Rat Jugular Vein." J. Appl. Physiol. 36: 391-2. https://doi.org/10.1152/jappl.1974.36.3.391.

[22] Matthews, D. R., Hosker, J. P., Rudenski, A. S., Naylor, B. A., Treacher, D. F., and Turner, R. C. 1985. "Homeostasis Model Assessment: Insulin Resistance and Beta-Cell Function from Fasting Plasma Glucose and Insulin Concentrations in Man.” Diabetologia 28: 412-9.

[23] Folch, J., Lees, M., and Sloane-Stanley, G. H. 2003. “A Simple Method for the Isolation and Purification of Total Lipids from Animal Tissues." J Biol Chem 226: 497-509.

[24] Bracht, A., Ishii-Iwamoto, E. L., and Salgueiro-Pagadigorria, C. L. 2003. "Técnica de centrifugação e fracionamento cellular." In Métodos de laboratório em bioquímica, edited by A. Bracht and E. L. Ishii-Iwamoto. São Paulo: Manole, 77-101.

[25] Tyler, D. D. 1975. "Polarographic Assay and Intracellular Distribution of Superoxide Dismutase in Rat Liver." Biochem. $\quad J . \quad$ 147: 493-504. https://doi.org/10.1042/bj1470493.

[26] Lowry, O. H., Rosebrough, N. J., Farr, A. L., and Randall, R. J. 1951. "Protein Measurement with the Folin Phenol Reagent.” J. Biol. Chem. 193: 265-75.

[27] Garland, P. B., Shepherd, D., Nicholls, D. G., Yates, D. W., and Light, P. A. 1969. "Interactions between Fatty Acid Oxidation and the Tricarboxylic Acid Cycle." In Citric Acid Cycle, edited by J. M. Lowestein. New York: Dekker, 163-212.

[28] Berson, A., De Beco, V., Lettéron, P., Robin, M. A., Moreau, C., El Kahwaji J., et al. 1998. "Steatohepatitis-Inducing Drugs Cause Mitochondrial Dysfunction and Lipid Peroxidation in Rat Hepatocytes." Gastroenterology 114: 764-74.

[29] Hissin, P. J., and Hilf, R. 1976. "A Fluorometric Method for Determination of Oxidized and Reduced Glutathione in Tissues." Anal Biochem 74: 214-26. https://doi.org/10.1016/0003-2697(76)90326-2.

[30] Paglia, E. D., and Valentine, W. N. 1967. "Studies on the Quantitative and Qualitative Characterization of Erythrocyte Glutatione Peroxidase." J. Lab. Clin. Med. 70: 158-69.

[31] Tian, W. N., Pignatare, N., and Stanton, R. C. 1994. "Signal Transduction Proteins That Associate with the Platelet-Derived Growth Factor (PDGF) Receptor Mediate the PDGF-Induced Release of Glucose-6-Phosphate Dehydrogenase from Permeabilized Cells.” J. Biol. Chem. 269: 14798-805.

[32] Sheeran, F. L., Rydstrom, J., Shakhparonov, M. I., Pestov, N. B., and Pepe, S. 2010. "Diminished NADPH 
Transhydrogenase Activity and Mitochondrial Redox Regulation in Human Failing Myocardium." Biochim. Biophys. Acta 1797: 1138-48. https://doi.org/10.1016/j.bbabio.2010.04.002.

[33] Ronchi, J. A., Figueira, T. R., Ravagnani, F. G., Oliveira, H. C. F., Vercesi, A. E., and Castilho, R. F. 2013. "A Spontaneous Mutation in the Nicotinamide Nucleotide Transhydrogenase Gene of c57bl/6j Mice Results in Mitochondrial Redox Abnormalities." Free Radic. Biol. Med. 63: 446-56. https://doi.org/10.1016/j.freeradbiomed.2013.05.049.

[34] Guarnier, F. A., Cecchini, A. L., Suzukawa, I. A., Maragno, A. L. G. C., Sima, A. A. N. C., Gomes, M. D., et al. 2010. "Time Course of Skeletal Muscle Loss and Oxidative Stress in Rats with Walker 256 Solid Tumor." Muscle Nerve 42: 950-8. https://doi.org/10.1002/mus.21798.

[35] Dalle-Donne, I., Rossi, R., Giustarini, D., Milzani, A., and Colombo, R. 2003. "Protein Carbonyl Groups as Biomarkers of Oxidative Stress." Clin. Chim. Acta 329 (1-2): 23-38. https://doi.org/10.1016/S0009-8981(03)00003-2.

[36] Harishekar, M. B., and Kiran, B. 2011. "Effect of Lead, Alcohol and Vitamin E on Protein Carbonyl Content in Rats." JAPS 01 (09): 154-6.

[37] Ohkawa, H., Ohishi, N., and Yagi, K. 1979. "Assay for Lipid Peroxides in Animal Tissues by Thiobarbituric Acid Reaction." Analyt Biochem 95: 351-8. https://doi.org/10.1016/0003-2697(79)90738-3.

[38] Sanchez-Muniz, F. J., and Bastida, S. 2008. "Do Not Use the Friedewald Formula to Calculate LDL-Cholesterol in Hypercholesterolaemic Rats." Eur. J. Lipid. Sci. Technol. 110: 295-301. https://doi.org/10.1002/ejlt.200700280.

[39] Winkler, G., Kiss, S., Keszthelyi, L., Sápi, Z., Ory, I., Salamon, F., et al. 2003. "Expression of Tumor Necrosis Factor (TNF)-Alpha Protein in the Subcutaneous and Visceral Adipose Tissue in Correlation with Adipocyte Cell Volume, Serum TNF-Alpha, Soluble Serum TNF-Receptor-2 Concentrations and C-Peptide Level." $\begin{array}{lllll}\text { Eur. J. } & \text { Endocrinol. } 149 & \text { (2): } 129-35 .\end{array}$ https://doi.org/10.1530/eje.0.1490129.

[40] Altomonte, J., Harbaran, S., Richter, A., and Dong, H. 2003. "Fat Depot-Specific Expression of Adiponectin Is Impaired in Zucker Fatty Rats." Metabolism 52: 958-63. http://dx.doi.org/10.1016/S0026-0495(03)00092-1.
[41] Gabriely, I., and Barzilai, N. 2003. "Surgical Removal of Visceral Adipose Tissue: Effects on Insulin Action." Curr. Diab. Rep. 3: 201-6.

[42] Diehl, A. M. 2004. "Tumor Necrosis Factor and Its Potential Role in Insulin Resistance and Nonalcoholic Fatty Liver Disease." Clin. Liver Dis. 8: 619-38. https://doi.org/10.1016/j.cld.2004.04.012.

[43] Jacobsson, B., and Smith, U. 1972. "Effect of Cell Size on Lipolysis and Antilipolytic Action of Insulin in Human Fat Cells." J. Lipid. Res. 13: 651-6.

[44] Franck, N., Stenkula, K. G., Ost, A., Lindstrom, T., Stralfors, P., and Nystron, F. H. 2007. "Insulin Induced GLUT4 Translocation to the Plasma Membrane Is Blunted in Large Compared with Small Primary Fat Cells Isolated from the Same Individual." Diabetologia 50 (8): 1716-22. https://doi.org/10.1007/s00125-007-0713-1.

[45] Han, S., Liang, C. P., Westerterp, M., Senokuchi, T., Welch, C. L., Wang, Q., et al. 2009. "Hepatic Insulin Signaling Regulates VLDL Secretion and Atherogenesis in Mice." J. Clin. Invest. 119: 1029-41. https://doi.org/10.1172/JCI36523.

[46] Sparks, J. D., Sparks, C. E., and Adeli, K. 2012. "Selective Hepatic Insulin Resistance, VLDL Overproduction, and Hypertriglyceridemia" Arterioscler. Thromb. Vasc. Biol. 32: 2104-12. https://doi.org/10.1161/ATVBAHA.111.241463.

[47] Barp, J., Araújo, A. R. S., Fernandes, T. R. G., Rigatto, K. V., Liesuy, S., Belló-Klein, A., et al. 2002. "Myocardial Antioxidant and Oxidative Stress Changes due to Sex Hormones." Braz. J. Med. Biol. Res. 35: 1075-81. http://dx.doi.org/10.1590/S0100-879X2002000900008.

[48] Borrás, C., Gambini, J., López-Grueso, R., Pallardó, F.V., and Viña, J. 2010. "Direct Antioxidant and Protective Effect of Estradiol on Isolated Mitochondria." Biochim. Biophys. Acta 1802: 205-11. https://doi.org/10.1016/j.bbadis.2009.09.007.

[49] Rydstrom, J. $2006 . \quad$ "Mitochondrial Transhydrogenase-A Key Enzyme in Insulin Secretion and, Potentially, Diabetes." Trends. Biochem. Sci. 31 (7): 355-8. https://doi.org/10.1016/j.tibs.2006.05.003.

[50] Freeman, H., Shimomura, K., Cox, R. D., and Ashcroft, F. M. 2006. "Nicotinamide Nucleotide Transhydrogenase: A Link between Insulin Secretion, Glucose Metabolism and Oxidative Stress." Biochem. Soc. Trans. 34 (5): 806-10. https://doi.org/10.1042/BST0340806. 